\title{
Effects of weighted baseball throwing during warm-up on ball velocity and upper extremity muscle activation in baseball pitchers
}

\author{
Yun-A Shin', Won-Ho Choi ${ }^{2 * *}$ \\ 'Departments of Prescription \& Rehabilitation of Exercise College of Physical Exercise and Kinesiologic Medical Science, Graduate School Dankook University, Cheonan, \\ Korea \\ 2Department of Physical Education, Graduate School Dankook University, Yongin, Korea
}

The objective of this study was to investigate the changes in the muscle activation of high school and college baseball pitchers during throwing of the ball with maximum effort (TBME) using a regular baseball (RB) subsequent to using a light baseball (LB), RB, and overweight baseball $(\mathrm{OB})$ during warm-up (WU) and the resulting changes in the pitch velocity. The study aimed to use the findings in providing basic data for a training program designed to increase the pitch velocity of baseball pitchers. The study population consisted of 12 high school and college baseball players. The study measured and analyzed the upper extremity muscle activation and ball velocity in the stride, arm cocking, and acceleration phases during TBME using an RB subsequent to using an $L B, R B$, and $O B$ during WU. During WU, the ball velocity was higher when pitching with an $\mathrm{LB}$ than with an $\mathrm{RB}$ or $\mathrm{OB}$ and when pitching with an $\mathrm{RB}$ than with an $\mathrm{OB}$. However, there were no significant differences in the ball velocity when pitching with an RB during TBME. In conclusion, WU using weighted baseballs resulted in varying muscle activations, and although the velocity decreased when pitching with an $O B$, no difference was found during TBME using an RB. Therefore, it is believed that using weighted baseballs during WU does not have an effect on the ball velocity during TBME; future studies are needed on the effects through long-term training.

Keywords: Pitcher, Ball velocity, Weighted baseball, Muscle activity

\section{INTRODUCTION}

In a baseball game, pitchers play a key role in determining the outcome of the game, while the velocity and accuracy of pitches thrown by the pitchers are considered to be very important factors (Yang et al., 2013). The ability to throw a ball at a high velocity requires a strong upper extremity muscle and a high power to generate maximum muscle strength within a short timeframe during the pitching motion (Potteiger et al., 1989).

Many precedent studies have reported that training with light and heavy balls can help increase the pitch velocity (DeRenne et al., 1994; DeRenne and Szymanski, 2009; Escamilla et al., 2000; Pavlovich, 2014); not only amateur players who are starting their training, but also even major league baseball teams have developed, and applied training methods based on weighted balls (Cressey, 2009; DriveLine Baseball, 2011).

When throwing a ball, the force-time relationship is formed within a very short time of approximately $0.15 \mathrm{sec}$, i.e., from the moment the foot touches the ground to the moment the ball leaves the hand (Escamilla et al., 1998). Therefore, it is necessary to increase the power through training to overcome resistance while also generating the fastest muscle contraction within the shortest time possible and consequently to increase the pitch velocity (Fleisig et al., 1996). A lighter ball allows the body segments to move faster while requiring less force from the muscles, whereas a heavier ball requires greater force from the muscles while slowing the movement of the body segments (Escamilla et al., 2000). Consequently, training with lighter balls increases arm speed, whereas training
*Corresponding author: Won-Ho Choi (iD https://orcid.org/0000-0002-7575-9543 Department of Physical Education, Graduate School Dankook University, 152 Jukjeon-ro, Suji-gu, Yongin 16890, Korea

Tel: +82-2-3436-1307, Fax: +82-2-446-0810, E-mail: yoonchan45@naver.com Received: March 10, 2018 / Accepted: April 28, 2018
This is an Open Access article distributed under the terms of the Creative Commons Attribution Non-Commercial License (http://creativecommons.org/licenses/by-nc/4.0/) which permits unrestricted non-commercial use, distribution, and reproduction in any medium, provided the original work is properly cited. 
with heavier balls increases arm muscle strength (DeRenne and Szymanski, 2009; Escamilla et al., 2000).

Such training programs include using balls with weights within 20\% (4-6 oz) of a regular baseball (RB, 5 oz) employed when pitching from the mound, as well as throwing and catching light baseball (LB), and overweight baseball (OB) on a flat ground (DriveLine Baseball, 2011; House, 2016). DeRenne et al. (1985) and DeRenne et al. (1990) conducted 10 weeks of pitching training with 4- to 6-oz baseballs on high school pitchers and reported that the groups that used an LB and OB showed an increased pitch velocity. Escamilla et al. (2000) also reported that 10 weeks of training with 4- to 4.72-oz LB and 5.25- to 17-oz OB resulted in a $3.20 \%$ increase in the pitch velocity, showing no difference in the ball velocity after training with different weighted balls.

However, in an acute experiment in which young high school and college pitchers were asked to throw 10 balls with maximal effort, increase in the ball weight resulted in decreased torque and force in the elbow and shoulder joint and decreased ball velocity; further, using an LB was effective in increasing the ball velocity and reducing kinetics (Fleisig et al., 2017). Moreover, the study also reported that using an RB (5 oz) showed higher shoulder and elbow joint torques than using an LB ( $4 \mathrm{oz})$ and that kinetics did not change according to the weight of the ball (Fleisig et al., 2006); however, overweight warm-up (WU; $11 \mathrm{oz}$ ) increased the velocity and accuracy of an RB, i.e., the ball velocity increased by $5 \%-10 \%$ (Brose and Hanson, 1967; Van Huss et al., 2013). Therefore, acute studies on the changes in ball velocity according to the weight of the ball have reported inconsistent results.

Most precedent studies have reported on the changes in the angular velocity of the joints according to the weight of the ball through motion analyses during throwing motions; however, studies analyzing muscle activation related to the force generated when throwing a ball are still lacking. Therefore, examination of the changes in the responses of the muscles related to throwing an RB versus baseballs with different weights is needed to provide accurate information on the association between the changes in the activation of the muscles according to the weight of the ball and the resulting changes in the pitch velocity.

Accordingly, the objective of this study was to investigate the changes in the muscle activation of high school and college baseball pitchers during throwing of the ball with maximum effort (TBME) using an $\mathrm{RB}$ subsequent to using an $\mathrm{LB}, \mathrm{RB}$, and $\mathrm{OB}$ during $\mathrm{WU}$ and the resulting changes in the pitch velocity. The study aimed to use the findings in providing basic data for a training program designed to increase the pitch velocity of baseball pitchers.

\section{MATERIALS AND METHODS}

\section{Study population}

The study population consisted of 12 pitchers from Jangan High School in Suwon city and Dankook University in Cheonan city. All pitchers were first year students with at least 5 years of pitching experience and were overhand pitchers with no history of elbow or shoulder injury. The physical characteristics of the subjects are shown in Table 1.

\section{Muscle activation test}

\section{Electromyography electrodes attachment}

The activation of the muscles active during the exercise load test was measured using a wireless electromyography (EMG) device. The skin was cleaned using alcohol to remove any foreign substances prior to attachment of a total of eight electrodes (distance between centers: $1.5 \mathrm{~cm}$ ). Subsequently, the electrodes were attached to the internal and external rotator muscles (upper trapezius, deltoid, supraspinatus, infraspinatus, pectoralis minor, and serratus anterior muscles) and the shoulder muscles (biceps brachii andtriceps brachii muscles) on the dominant side (right side). Prior to the experiment, each subject underwent practice for measuring the maximal voluntary isometric contraction (MVIC) in each of the eight muscles and was provided enough rest prior to the actual measurement. The electrode attachment locations of the muscles and MVIC measurement followed the guidelines presented in precedent studies (Cram et al., 1998) and the EMG manufacturer's protocol (SENIAM Guide Line).

\section{MVIC measurement}

For the MVIC measurement, data were collected by having the eight muscles mentioned above perform a specific motion for 5 sec. Theupper trapezius and supraspinatus muscles were measured by lowering the arm downward and having the shoulder joint ex-

Table 1. The characteristic of pitchers $(n=12)$

\begin{tabular}{lr}
\hline Characteristic & Mean \pm SD \\
\hline Age $(\mathrm{yr})$ & $20.2 \pm 1.64$ \\
Height $(\mathrm{cm})$ & $181.0 \pm 3.08$ \\
Weight $(\mathrm{kg})$ & $84.8 \pm 6.76$ \\
Body mass index $\left(\mathrm{kg} / \mathrm{m}^{2}\right)$ & $25.86 \pm 1.45$ \\
Career $(\mathrm{yr})$ & $10.8 \pm 2.17$
\end{tabular}

SD, standard deviation. 
ert maximum force towards the ear. The anterior deltoid muscle was measured by erecting the upper body in a straight line and exerting maximum force with the elbow extended to the side in a 90 degreedirection. The infraspinatus muscle was measured by exerting maximum force while the shoulder performed external rotation towards the outside direction. The serratus anterior muscle was measured while the arm was raised at $90^{\circ}$ and pushed against the wall with maximum force. The latissimus dorsi muscle was measured with the upper body bent at $90^{\circ}$ and the elbow bent to form a $90^{\circ}$ angle between the forearm and upper arm while the elbow exerted maximum force towards the abdomen. The biceps brachii muscle was measured with the elbow maintaining a $120^{\circ}$ angle while the forearm exerted maximum force towards the upper arm. The triceps brachii muscle was measured with the elbow maintaining a $90^{\circ}$ angle while the forearm exerted maximum force in the direction opposite of the upper arm.

\section{Measurement of the muscle activation during pitching motion}

Prior to pitching, the pitchers prepared for TBME by performing WU exercises and toss-and-catch for 10-15 min. LB (4 oz), $\mathrm{RB}(5 \mathrm{oz})$, and $\mathrm{OB}(8 \mathrm{oz})$ were used during WU, where 10 pitches were performed at 70\%-85\% of TBME; thereafter, another 10 pitches were performed using an RB at $100 \%$ of TBME. Between

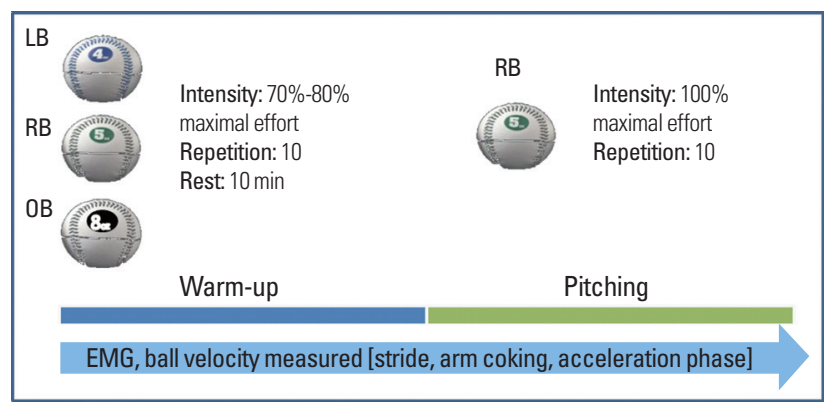

Fig. 1. Experimental design. LB, lighted baseball (4 oz); RB, regular baseball (5 $\mathrm{oz}) ; \mathrm{OB}$, overweight baseball (8 oz); EMG, electromyography. pitching with different weighted balls, enough rest periods of 10 min was provided (Fig. 1).

Muscle activation was measured during WU and TBME. The bandwidth of the EMG signals was filtered using a high-pass filter of $10 \mathrm{~Hz}$ and a low-pass filter of $350 \mathrm{~Hz}$, followed by full-wave rectification. To synchronize the EMG data, 6-mm high-speed digital video cameras were installed to acquire images of the pitching motions and the images and EMG data were synchronized and analyzed. The baseball pitching motion was divided into six phases, with the phases divided by camera analysis. For the analysis of the muscle activation, only the stride, arm cocking, and arm acceleration phases, where the shoulder muscles are highly recruited, were selected and analyzed.

\section{Pitch velocity measurement}

For the measurement of the pitch velocity, each subject performed enough WU exercises $(20 \mathrm{~min})$ and toss-and-catch $(\geq 20$ balls). Subsequently, each subject pitched from the mound to the home plate (distance of $18.44 \mathrm{~m}$ ), and a radar gun (Sport radar, 24.7 GHZ, SP78585, Applied Concepts, Inc., Northbrook, IL, USA) was used to measure the velocity of the fastballs that were thrown for the strikes.

\section{Static analysis}

The study data were analyzed using IBM SPSS ver. 18.0 (IBM Co., Armonk, NY, USA). All measured values were expressed as means and standard deviations. Two-way repeated measures analysis of variance (ANOVA) was used to analyze the changes in the pitch velocity and muscle activation according to the weight of the baseball, while the least significant difference method was used for the post hoc analysis. One-way ANOVA was used to analyze the changes in the muscle activation according to the weight of the baseball. The correlation between the muscle activation and ball velocity was analyzed via Pearson correlation analysis using the highest muscle activation throughout all phases and the highest

Table 2. The changes of ball velocity

\begin{tabular}{|c|c|c|c|c|c|}
\hline Condition & Warm-up & Maximum effort & \multicolumn{2}{|c|}{$F$} & Sig \\
\hline $\mathrm{LB} \rightarrow \mathrm{RB}$ & $121.00 \pm 4.05^{a), *, b), * * *}$ & $121.58 \pm 4.03$ & $\mathrm{~T}$ & 37.982 & 0.000 \\
\hline $\mathrm{RB} \rightarrow \mathrm{RB}$ & $119.83 \pm 4.45^{c l * * * *}$ & $120.92 \pm 4.36$ & C & 13.646 & 0.001 \\
\hline $\mathrm{OB} \rightarrow \mathrm{RB}$ & $85.91 \pm 7.16$ & $121.75 \pm 3.19$ & $\mathrm{~T} \times \mathrm{C}$ & 39.774 & 0.000 \\
\hline Posthoc & $F=157.966 . \mathrm{Sig}=0.000$ & $F=0.138, \operatorname{Sig}=0.871$ & & & \\
\hline
\end{tabular}

Values are presented as mean \pm standard deviation.

$\mathrm{LB}$, light baseball; $\mathrm{RB}$, regular baseball; $\mathrm{OB}$, overweight baseball.

${ }^{a}$ The difference of light vs. regular baseball pitching. ${ }^{\mathrm{b} T}$ The difference of light vs. overweigh baseball pitching. ${ }^{\mathrm{I} T h e ~ d i f f e r e n c e ~ o f ~ r e g u l a r ~ v s . ~ o v e r w e i g h t ~ b a s e b a l l ~ p i t c h i n g . ~}$

${ }^{*} P<0.05$. ${ }^{* *} P<0.001$. 
ball velocity. The statistical significance level was set at $\alpha=0.05$.

\section{RESULTS}

Changes in the ball velocity according to the weight of the baseball

The changes in the ball velocity during TBME using an RB subsequent to using the weighted baseballs are shown in Table 2 . There was a significant difference in the ball velocity between
WU and TBME $(P<0.001)$, while significant differences according to the use of weighted balls $(P<0.01)$ and interaction effects according to TBME and the weighted ball conditions $(P<0.001)$ were also found. In the post hoc test, the ball velocity was higher when pitching with an $\mathrm{LB}$ during WU than with an $\mathrm{RB}(P<0.05)$ and $\mathrm{OB}(P<0.001)$. During TBME, pitching with an RB subsequent to pitching with an $\mathrm{LB}$ and $\mathrm{OB}$ resulted in a higher ball velocity; however, the differences were not statistically significant.

Table 3. Muscle activity during stride phase

\begin{tabular}{|c|c|c|c|c|c|c|}
\hline \multirow{2}{*}{$\begin{array}{l}\text { Muscle } \\
\text { UT ( } \mu \mathrm{V}, \% \mathrm{MVIC})\end{array}$} & \multirow{2}{*}{$\begin{array}{l}\text { Condition } \\
\mathrm{LB} \rightarrow \mathrm{RB}\end{array}$} & \multirow{2}{*}{$\begin{array}{c}\text { Warm-up } \\
76.81 \pm 60.28\end{array}$} & \multirow{2}{*}{$\begin{array}{c}\text { Maximum effort } \\
90.85 \pm 61.98\end{array}$} & \multicolumn{2}{|c|}{$F$} & \multirow{2}{*}{$\begin{array}{r}\text { Sig } \\
0.848\end{array}$} \\
\hline & & & & $\mathrm{T}$ & 0.037 & \\
\hline & $\mathrm{RB} \rightarrow \mathrm{RB}$ & $97.93 \pm 67.71$ & $96.08 \pm 45.14$ & C & 0.239 & 0.789 \\
\hline & $\mathrm{OB} \rightarrow \mathrm{RB}$ & $105.99 \pm 48.82$ & $86.75 \pm 63.48$ & $\mathrm{~T} \times \mathrm{C}$ & 0.623 & 0.544 \\
\hline Posthoc & & $F=0.360$, Sig $=0.701$ & $F=0.066$, Sig $=0.936$ & & & \\
\hline \multirow[t]{3}{*}{$\mathrm{SP}(\mu \mathrm{V}, \% \mathrm{MVIC})$} & $\mathrm{LB} \rightarrow \mathrm{RB}$ & $95.75 \pm 46.34$ & $113.17 \pm 46.89$ & T & 0.576 & 0.454 \\
\hline & $\mathrm{RB} \rightarrow \mathrm{RB}$ & $116.87 \pm 33.89$ & $113.79 \pm 46.44$ & C & 3.914 & 0.032 \\
\hline & $\mathrm{OB} \rightarrow \mathrm{RB}$ & $139.64 \pm 33.44^{a \mid, *, b, t}$, & $145.79 \pm 28.78$ & $\mathrm{~T} \times \mathrm{C}$ & 0.434 & 0.652 \\
\hline Posthoc & & $F=3.274, \operatorname{Sig}=0.053$ & $F=2.014$, Sig $=0.153$ & & & \\
\hline \multirow[t]{3}{*}{ IF $(\mu \mathrm{V}, \% \mathrm{MVIC})$} & $\mathrm{LB} \rightarrow \mathrm{RB}$ & $41.94 \pm 32.45$ & $62.37 \pm 49.28$ & $\mathrm{~T}$ & 9.702 & 0.004 \\
\hline & $\mathrm{RB} \rightarrow \mathrm{RB}$ & $37.13 \pm 18.80$ & $52.84 \pm 38.07$ & C & 0.304 & 0.740 \\
\hline & $\mathrm{OB} \rightarrow \mathrm{RB}$ & $26.48 \pm 9.55$ & $58.78 \pm 43.46$ & $\mathrm{~T} \times \mathrm{C}$ & 0.454 & 0.640 \\
\hline Posthoc & & $F=1.609, \mathrm{Sig}=0.219$ & $F=0.120, S i g=0.887$ & & & \\
\hline \multirow[t]{3}{*}{$\mathrm{SA}(\mu \mathrm{V}, \% \mathrm{MVIC})$} & $\mathrm{LB} \rightarrow \mathrm{RB}$ & $24.34 \pm 11.60$ & $38.75 \pm 35.82$ & $\mathrm{~T}$ & 0.362 & 0.552 \\
\hline & $\mathrm{RB} \rightarrow \mathrm{RB}$ & $34.41 \pm 28.41$ & $25.25 \pm 9.18$ & C & 0.079 & 0.924 \\
\hline & $\mathrm{OB} \rightarrow \mathrm{RB}$ & $30.84 \pm 16.26$ & $35.38 \pm 28.10$ & $\mathrm{~T} \times \mathrm{C}$ & 1.590 & 0.223 \\
\hline Posthoc & & $F=0.702, \operatorname{Sig}=0.504$ & $F=0.635, \operatorname{Sig}=0.538$ & & & \\
\hline \multirow[t]{3}{*}{$\mathrm{AD}(\mu \mathrm{V}, \% \mathrm{MVIC})$} & $\mathrm{LB} \rightarrow \mathrm{RB}$ & $91.13 \pm 56.10$ & $99.6 \pm 61.30$ & $\mathrm{~T}$ & 0.019 & 0.891 \\
\hline & $\mathrm{RB} \rightarrow \mathrm{RB}$ & $91.79 \pm 66.96$ & $85.74 \pm 69.71$ & C & 0.570 & 0.572 \\
\hline & $\mathrm{OB} \rightarrow \mathrm{RB}$ & $116.78 \pm 56.79$ & $109.84 \pm 51.56$ & $\mathrm{~T} \times \mathrm{C}$ & 0.220 & 0.804 \\
\hline Posthoc & & $F=0.482, \operatorname{Sig}=0.623$ & $F=0.411$, Sig $=0.667$ & & & \\
\hline \multirow[t]{3}{*}{ LD ( $\mu \mathrm{V}, \% \mathrm{MVIC})$} & $\mathrm{LB} \rightarrow \mathrm{RB}$ & $44.69 \pm 43.97$ & $47.38 \pm 43.07$ & T & 1.792 & 0.192 \\
\hline & $\mathrm{RB} \rightarrow \mathrm{RB}$ & $39.58 \pm 44.69$ & $66.90 \pm 95.59$ & C & 0.229 & 0.797 \\
\hline & $\mathrm{OB} \rightarrow \mathrm{RB}$ & $29.93 \pm 31.96$ & $50.23 \pm 49.74$ & $\mathrm{~T} \times \mathrm{C}$ & 0.342 & 0.713 \\
\hline Posthoc & & $F=0.340$, Sig $=0.715$ & $F=0.248, S i g=0.782$ & & & \\
\hline \multirow[t]{3}{*}{$\mathrm{BB}(\mu \mathrm{V}, \% \mathrm{MVIC})$} & $\mathrm{LB} \rightarrow \mathrm{RB}$ & $42.26 \pm 26.26$ & $83.69 \pm 61.17$ & $\mathrm{~T}$ & 0.425 & 0.520 \\
\hline & $\mathrm{RB} \rightarrow \mathrm{RB}$ & $76.54 \pm 53.34$ & $59.92 \pm 41.49$ & C & 1.270 & 0.297 \\
\hline & $\mathrm{OB} \rightarrow \mathrm{RB}$ & $90.58 \pm 53.32$ & $87.54 \pm 52.21$ & $\mathrm{~T} \times \mathrm{C}$ & 2.478 & 0.103 \\
\hline Posthoc & & $F=1.479, \mathrm{Sig}=0.246$ & $F=0.610$, Sig $=0.551$ & & & \\
\hline \multirow[t]{3}{*}{$\mathrm{TB}(\mu \mathrm{V}, \% \mathrm{MVIC})$} & $\mathrm{LB} \rightarrow \mathrm{RB}$ & $53.45 \pm 54.39$ & $77.44 \pm 61.38$ & $\mathrm{~T}$ & 1.499 & 0.231 \\
\hline & $\mathrm{RB} \rightarrow \mathrm{RB}$ & $67.60 \pm 59.40$ & $78.67 \pm 74.28$ & C & 0.507 & 0.608 \\
\hline & $\mathrm{OB} \rightarrow \mathrm{RB}$ & $35.87 \pm 38.01$ & $36.15 \pm 44.58$ & $\mathrm{~T} \times \mathrm{C}$ & 1.532 & 0.234 \\
\hline Posthoc & & $F=1.440, \mathrm{Sig}=0.255$ & $F=2.009, \operatorname{Sig}=0.154$ & & & \\
\hline
\end{tabular}

Values are presented as mean \pm standard deviation.

MVIC, maximal voluntary isometric contraction; LB, light baseball; RB, regular baseball; OB, overweight baseball; UT, upper trapezius; SP, supraspinatus; IF, infraspinatus; SA, serratus anterior; $A D$, anterior deltoid; $L D$, latissimus dorsi; $B I$, bicephalus brachii; $T R$, triceps brachii.

a'The difference of light vs. overweigh baseball pitching. ${ }^{b}$ The difference of regular vs. overweight baseball pitching.

${ }^{*} P<0.05$. ${ }^{\top}$ The trend of significance. 
Table 4. Muscle activity during arm coking phase

\begin{tabular}{|c|c|c|c|c|c|c|}
\hline \multirow{2}{*}{$\begin{array}{l}\text { Muscle } \\
\text { UT ( } \mu \mathrm{V}, \% \mathrm{MVIC})\end{array}$} & \multirow{2}{*}{$\begin{array}{l}\text { Condition } \\
\mathrm{LB} \rightarrow \mathrm{RB}\end{array}$} & \multirow{2}{*}{$\begin{array}{c}\text { Warm-up } \\
94.64 \pm 62.77\end{array}$} & \multirow{2}{*}{$\begin{array}{c}\text { Maximum effort } \\
109.05 \pm 65.27\end{array}$} & \multicolumn{2}{|c|}{$F$} & \multirow{2}{*}{$\begin{array}{c}\text { Sig } \\
0.577\end{array}$} \\
\hline & & & & T & 0.319 & \\
\hline & $\mathrm{RB} \rightarrow \mathrm{RB}$ & $100.97 \pm 74.40$ & $92.00 \pm 71.41$ & C & 0.426 & 0.658 \\
\hline & $\mathrm{OB} \rightarrow \mathrm{RB}$ & $94.3 \pm 75.40$ & $65.75 \pm 45.45$ & $\mathrm{~T} \times \mathrm{C}$ & 0.830 & 0.447 \\
\hline Posthoc & & $F=0.028, \mathrm{Sig}=0.972$ & $F=1.249, \mathrm{Sig}=0.303$ & & & \\
\hline \multirow[t]{3}{*}{$\mathrm{SP}(\mu \mathrm{V}, \% \mathrm{MVIC})$} & $\mathrm{LB} \rightarrow \mathrm{RB}$ & $102.49 \pm 61.74$ & $88.10 \pm 46.48$ & T & 0.198 & 0.660 \\
\hline & $\mathrm{RB} \rightarrow \mathrm{RB}$ & $99.51 \pm 54.59$ & $102.06 \pm 50.01$ & C & 0.278 & 0.759 \\
\hline & $\mathrm{OB} \rightarrow \mathrm{RB}$ & $86.62 \pm 54.47$ & $85.21 \pm 46.94$ & $\mathrm{~T} \times \mathrm{C}$ & 0.265 & 0.769 \\
\hline Posthoc & & $F=0.219, \operatorname{Sig}=0.805$ & $F=0.355, \operatorname{Sig}=0.705$ & & & \\
\hline \multirow[t]{3}{*}{$\mathrm{IF}(\mu \mathrm{V}, \% \mathrm{MVIC})$} & $\mathrm{LB} \rightarrow \mathrm{RB}$ & $80.92 \pm 47.67$ & $101.07 \pm 61.14$ & T & 0.015 & 0.902 \\
\hline & $\mathrm{RB} \rightarrow \mathrm{RB}$ & $88.70 \pm 57.40$ & $82.21 \pm 38.03$ & C & 0.092 & 0.913 \\
\hline & $\mathrm{OB} \rightarrow \mathrm{RB}$ & $89.26 \pm 50.29$ & $80.09 \pm 32.43$ & $\mathrm{~T} \times \mathrm{C}$ & 0.600 & 0.556 \\
\hline Posthoc & & $F=0.081$, Sig $=0.923$ & $F=0.562, S i g=0.577$ & & & \\
\hline \multirow[t]{3}{*}{$\mathrm{SA}(\mu \mathrm{V}, \% \mathrm{MVIC})$} & $\mathrm{LB} \rightarrow \mathrm{RB}$ & $79.93 \pm 51.46$ & $74.68 \pm 52.03$ & T & 1.286 & 0.267 \\
\hline & $\mathrm{RB} \rightarrow \mathrm{RB}$ & $92.62 \pm 64.71$ & $79.62 \pm 48.02$ & C & 0.088 & 0.916 \\
\hline & $\mathrm{OB} \rightarrow \mathrm{RB}$ & $93.84 \pm 60.47$ & $77.02 \pm 74.75$ & $\mathrm{~T} \times \mathrm{C}$ & 0.109 & 0.897 \\
\hline Posthoc & & $F=0.543$, Sig $=0.587$ & $F=0.017, \operatorname{Sig}=0.983$ & & & \\
\hline \multirow[t]{3}{*}{$\mathrm{AD}(\mu \mathrm{V}, \% \mathrm{MVIC})$} & $\mathrm{LB} \rightarrow \mathrm{RB}$ & $91.93 \pm 47.3^{\mathrm{al|} . *}$ & $136.64 \pm 18.90$ & T & 5.170 & 0.031 \\
\hline & $\mathrm{RB} \rightarrow \mathrm{RB}$ & $132.36 \pm 42.69$ & $155.43 \pm 29.44$ & C & 4.019 & 0.030 \\
\hline & $\mathrm{OB} \rightarrow \mathrm{RB}$ & $128.38 \pm 40.50$ & $125.87 \pm 21.88$ & $\mathrm{~T} \times \mathrm{C}$ & 2.034 & 0.150 \\
\hline Posthoc & & $F=3.942$, Sig $=0.031$ & $F=1.778, S i g=0.188$ & & & \\
\hline \multirow[t]{3}{*}{$\mathrm{LD}(\mu \mathrm{V}, \% \mathrm{MVIC})$} & $\mathrm{LB} \rightarrow \mathrm{RB}$ & $76.69 \pm 69.99$ & $128.35 \pm 50.63$ & T & 4.664 & 0.040 \\
\hline & $\mathrm{RB} \rightarrow \mathrm{RB}$ & $107.5 \pm 55.03$ & $94.21 \pm 62.42$ & C & 0.169 & 0.846 \\
\hline & $\mathrm{OB} \rightarrow \mathrm{RB}$ & $99.68 \pm 38.78$ & $124.18 \pm 32.05$ & $\mathrm{~T} \times \mathrm{C}$ & 3.767 & 0.036 \\
\hline Posthoc & & $F=1.390, \mathrm{Sig}=0.266$ & $F=0.419, \mathrm{Sig}=0.662$ & & & \\
\hline \multirow[t]{3}{*}{$\mathrm{BB}(\mu \mathrm{V}, \% \mathrm{MVIC})$} & $\mathrm{LB} \rightarrow \mathrm{RB}$ & $71.76 \pm 41.6$ & $71.88 \pm 25.26$ & $\mathrm{~T}$ & 1.994 & 0.169 \\
\hline & $\mathrm{RB} \rightarrow \mathrm{RB}$ & $97.57 \pm 61.47$ & $91.42 \pm 54.92$ & C & 0.756 & 0.479 \\
\hline & $\mathrm{OB} \rightarrow \mathrm{RB}$ & $63.75 \pm 40.93^{\mathrm{b} / * *}$ & $105.41 \pm 50.85$ & $\mathrm{~T} \times \mathrm{C}$ & 3.181 & 0.057 \\
\hline Posthoc & & $F=3.643, \mathrm{Sig}=0.040$ & $F=0.455, \mathrm{Sig}=0.639$ & & & \\
\hline \multirow[t]{3}{*}{$\mathrm{TB}(\mu \mathrm{V}, \% \mathrm{MVIC})$} & $\mathrm{LB} \rightarrow \mathrm{RB}$ & $76.89 \pm 47.46$ & $106.04 \pm 59.21$ & $T$ & 0.164 & 0.689 \\
\hline & $\mathrm{RB} \rightarrow \mathrm{RB}$ & $99.51 \pm 60.66$ & $92.85 \pm 58.39$ & C & 0.115 & 0.892 \\
\hline & $\mathrm{OB} \rightarrow \mathrm{RB}$ & $105.23 \pm 31.38$ & $96.96 \pm 66.76$ & $\mathrm{~T} \times \mathrm{C}$ & 1.086 & 0.352 \\
\hline Posthoc & & $F=0.120, S i g=0.887$ & $F=0.219, \mathrm{Sig}=0.805$ & & & \\
\hline
\end{tabular}

Values are presented as mean \pm standard deviation.

MVIC, maximal voluntary isometric contraction; LB, light baseball; RB, regular baseball; OB, overweight baseball; UT, upper trapezius; SP, supraspinatus; IF, infraspinatus; SA, serratus anterior; $A D$, anterior deltoid; $L D$, latissimus dorsi; $B I$, bicephalus brachii; $T R$, triceps brachii.

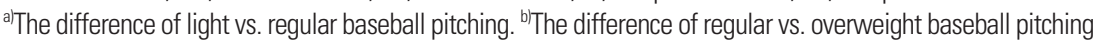

${ }^{*} P<0.05$.

\section{Changes in the muscle activation according to the weight of the baseball}

The changes in the muscle activation during TBME subsequent to WU and using weighted balls are shown in Tables 3-5. In the stride phase, the activation of the supraspinatus muscle showed differences according to the weighted ball conditions $(P<0.05)$, with pitching with an $\mathrm{OB}$ during WU showing a higher muscle activation than pitching with an $\mathrm{LB}(P<0.05)$ and $\mathrm{RB}(P=0.062)$. The activation of the infraspinatus muscle showed significant dif- ferences according to time $(P<0.05)$, with a higher muscle activation during TBME than during WU (Table 3).

In the arm cocking phase, the activation of the deltoid muscle showed significant differences according to time and the weighted ball conditions $(P<0.05)$, with a higher muscle activation when pitching with an RB than with an $\mathrm{LB}$ during WU $(P<0.05)$. The activation of the latissimus dorsi muscle also showed significant differences according to time during WU $(P<0.05)$ and the weighted ball conditions $(P<0.05)$. The activation of the biceps mus- 
Table 5. Muscle activity during acceleration phase

\begin{tabular}{|c|c|c|c|c|c|c|}
\hline \multirow{2}{*}{$\frac{\text { Muscle }}{\mathrm{UT}(\mu \mathrm{V}, \% \mathrm{MVIC})}$} & \multirow{2}{*}{$\begin{array}{l}\text { Condition } \\
\mathrm{LB} \rightarrow \mathrm{RB}\end{array}$} & \multirow{2}{*}{$\begin{array}{c}\text { Warm-up } \\
145.25 \pm 41.66\end{array}$} & \multirow{2}{*}{$\begin{array}{l}\text { Maximum effort } \\
114.54 \pm 69.33\end{array}$} & \multicolumn{2}{|c|}{$F$} & \multirow{2}{*}{$\begin{array}{l}\text { Sig } \\
0.273\end{array}$} \\
\hline & & & & $\mathrm{T}$ & 1.266 & \\
\hline & $\mathrm{RB} \rightarrow \mathrm{RB}$ & $157.73 \pm 59.13$ & $165.67 \pm 76.41$ & C & 0.402 & 0.533 \\
\hline & $\mathrm{OB} \rightarrow \mathrm{RB}$ & $118.25 \pm 48.71^{\mathrm{bl}, *}$ & $175.98 \pm 66.01$ & $T \times C$ & 8.355 & 0.008 \\
\hline Posthoc & & $F=2.616, \mathrm{Sig}=0.096$ & $F=3.456, \mathrm{Sig}=0.093$ & & & \\
\hline \multirow[t]{3}{*}{$\mathrm{SP}(\mu \mathrm{V}, \% \mathrm{MVIC})$} & $\mathrm{LB} \rightarrow \mathrm{RB}$ & $165.19 \pm 78.80$ & $161.04 \pm 59.15$ & $T$ & 2.552 & 0.124 \\
\hline & $\mathrm{RB} \rightarrow \mathrm{RB}$ & $174.74 \pm 92.67$ & $170.07 \pm 62.48$ & C & 0.171 & 0.683 \\
\hline & $\mathrm{OB} \rightarrow \mathrm{RB}$ & $122.97 \pm 53.24^{b \mid . *}$ & $159.81 \pm 47.70$ & $\mathrm{~T} \times \mathrm{C}$ & 2.271 & 0.146 \\
\hline Posthoc & & $F=4.311$, Sig $=0.062$ & $F=0.131$, Sig $=0.725$ & & & \\
\hline \multirow[t]{3}{*}{$\mathrm{IF}(\mu \mathrm{V}, \% \mathrm{MVIC})$} & $\mathrm{LB} \rightarrow \mathrm{RB}$ & $81.79 \pm 47.94$ & $90.22 \pm 45.91$ & T & 18.676 & 0.000 \\
\hline & $\mathrm{RB} \rightarrow \mathrm{RB}$ & $95.34 \pm 40.73$ & $113.42 \pm 91.73$ & C & 0.056 & 0.816 \\
\hline & $\mathrm{OB} \rightarrow \mathrm{RB}$ & $155.95 \pm 82.42^{a \mathrm{a}, * *, \mathrm{~b}) . *}$ & $146.55 \pm 94.71^{\mathrm{al}, *}$ & $\mathrm{~T} \times \mathrm{C}$ & 0.348 & 0.561 \\
\hline Posthoc & & $F=12.976, \operatorname{Sig}=0.004$ & $F=1.072, \operatorname{Sig}=0.023$ & & & \\
\hline \multirow[t]{3}{*}{$\mathrm{SA}(\mu \mathrm{V}, \% \mathrm{MVIC})$} & $\mathrm{LB} \rightarrow \mathrm{RB}$ & $72.85 \pm 37.24$ & $79.10 \pm 36.41$ & T & 7.766 & 0.011 \\
\hline & $\mathrm{RB} \rightarrow \mathrm{RB}$ & $69.11 \pm 31.10$ & $67.23 \pm 27.54$ & C & 0.027 & 0.872 \\
\hline & $\mathrm{OB} \rightarrow \mathrm{RB}$ & $107.73 \pm 68.62^{a, *, b, b}, *$ & $95.13 \pm 68.49$ & $\mathrm{~T} \times \mathrm{C}$ & 1.067 & 0.313 \\
\hline Posthoc & & $F=8.175, \mathrm{Sig}=0.016$ & $F=1.118, \operatorname{Sig}=0.315$ & & & \\
\hline \multirow[t]{3}{*}{$\mathrm{AD}(\mu \mathrm{V}, \% \mathrm{MVIC})$} & $\mathrm{LB} \rightarrow \mathrm{RB}$ & $145.22 \pm 43.40$ & $196.04 \pm 74.30$ & $\mathrm{~T}$ & 3.181 & 0.088 \\
\hline & $\mathrm{RB} \rightarrow \mathrm{RB}$ & $187.34 \pm 87.96$ & $201.72 \pm 85.17$ & C & 5.064 & 0.035 \\
\hline & $\mathrm{OB} \rightarrow \mathrm{RB}$ & $111.48 \pm 35.95^{b, *}$ & $176.18 \pm 71.38$ & $\mathrm{~T} \times \mathrm{C}$ & 0.213 & 0.600 \\
\hline Posthoc & & $F=3.696, \mathrm{Sig}=0.081$ & $F=1.593, \mathrm{Sig}=0.236$ & & & \\
\hline \multirow[t]{3}{*}{$\mathrm{LD}(\mu \mathrm{V}, \% \mathrm{MVIC})$} & $\mathrm{LB} \rightarrow \mathrm{RB}$ & $130.31 \pm 60.64$ & $197.61 \pm 186.41$ & $T$ & 5.853 & 0.020 \\
\hline & $\mathrm{RB} \rightarrow \mathrm{RB}$ & $150.46 \pm 80.43$ & $120.63 \pm 80.58$ & C & 1.555 & 0.225 \\
\hline & $\mathrm{OB} \rightarrow \mathrm{RB}$ & $69.40 \pm 47.53^{\mathrm{a}, . * * *, b), * *}$ & $136.74 \pm 77.03$ & $\mathrm{~T} \times \mathrm{C}$ & 0.000 & 0.999 \\
\hline Posthoc & & $F=24.936, \mathrm{Sig}=0.000$ & $F=1.512, \operatorname{Sig}=0.247$ & & & \\
\hline \multirow[t]{3}{*}{$\mathrm{BB}(\mu \mathrm{V}, \% \mathrm{MVIC})$} & $\mathrm{LB} \rightarrow \mathrm{RB}$ & $117.96 \pm 156.66$ & $131.26 \pm 158.21$ & $\mathrm{~T}$ & 1.885 & 0.184 \\
\hline & $\mathrm{RB} \rightarrow \mathrm{RB}$ & $123.29 \pm 125.44$ & $128.85 \pm 146.73$ & C & 0.002 & 0.963 \\
\hline & $\mathrm{OB} \rightarrow \mathrm{RB}$ & $100.24 \pm 169.48$ & $88.73 \pm 44.12$ & $\mathrm{~T} \times \mathrm{C}$ & 0.320 & 0.578 \\
\hline Posthoc & & $F=2.230, \mathrm{Sig}=0.163$ & $F=0.098$, Sig $=0.907$ & & & \\
\hline \multirow[t]{3}{*}{$\mathrm{TB}(\mu \mathrm{V}, \% \mathrm{MVIC})$} & $\mathrm{LB} \rightarrow \mathrm{RB}$ & $112.73 \pm 62.23$ & $134.34 \pm 72.93$ & $\mathrm{~T}$ & 15.182 & 0.001 \\
\hline & $\mathrm{RB} \rightarrow \mathrm{RB}$ & $115.14 \pm 72.08$ & $131.69 \pm 62.27$ & C & 1.147 & 0.296 \\
\hline & $\mathrm{OB} \rightarrow \mathrm{RB}$ & $71.70 \pm 48.45^{\mathrm{a}, * * * \mathrm{~b}), *}$ & $107.12 \pm 56.02$ & $\mathrm{~T} \times \mathrm{C}$ & 0.622 & 0.439 \\
\hline Posthoc & & $F=16.385, \operatorname{Sig}=0.002$ & $F=0.428, \mathrm{Sig}=0.656$ & & & \\
\hline
\end{tabular}

Values are presented as mean \pm standard deviation.

MVIC, maximal voluntary isometric contraction; LB, light baseball; RB, regular baseball; OB, overweight baseball; UT, upper trapezius; SP, supraspinatus; IF, infraspinatus; SA, serratus anterior; $A D$, anterior deltoid; $L D$, latissimus dorsi; $B I$, bicephalus brachii; $T R$, triceps brachii.

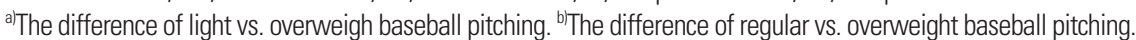

${ }^{*} P<0.05 .{ }^{* *} P<0.01 .{ }^{* *} P<0.001$.

cle showed significant differences according to time and the weighted ball conditions $(P<0.05)$, with a higher muscle activation when pitching with an $\mathrm{RB}$ than with an $\mathrm{OB}$ during $\mathrm{WU}(P<0.05)$ (Table 4).

In the arm acceleration phase, the upper trapezius muscle showed a lower activation when pitching with an $\mathrm{OB}$ than with an $\mathrm{RB}$ during WU $(P<0.05)$ and a significant interaction effect between time and the weighted ball conditions $(P<0.01)$. The supraspinatus muscle tended to show a lower activation when pitching with an $\mathrm{OB}$ than with an $\mathrm{RB}$ during WU $(P=0.062)$. The infraspinatus muscle showed a higher activation when pitching with an $\mathrm{OB}$ than with an $\mathrm{LB}(P<0.01)$ or $\mathrm{RB}(P<0.05)$ during WU $(P<0.05)$, while pitching with an $\mathrm{OB}$ showed a higher muscle activation than pitching with an LB during TBME $(P<0.05)$. Moreover, a significant difference was found according to time $(P<0.001)$, and the muscle activation was higher during TBME than during WU. The serratus anterior muscle showed a higher activation when pitching with an $\mathrm{OB}$ than with an $\mathrm{LB}$ or $\mathrm{RB}(P<0.05)$, while also 
Table 6. The relationship between ball velocity and muscle activity

\begin{tabular}{lc} 
Muscle activity & Ball velocity $(r)$ \\
\hline UT & $0.497^{*}$ \\
SP & 0.188 \\
IF & 0.118 \\
SA & -0.198 \\
AD & $0.466^{*}$ \\
LD & $0.423^{*}$ \\
BI & -0.057 \\
TR & $0.460^{*}$ \\
\hline
\end{tabular}

UT, upper trapezius; SP, supraspinatus; IF, infraspinatus; $S A$, serratus anterior; $A D$, anterior deltoid; $\mathrm{LD}$, latissimus dorsi; $\mathrm{Bl}$, bicephalus brachii; $\mathrm{TR}$, triceps brachii. ${ }^{*} P<0.05$.

showing significant differences according to time $(P<0.05)$.

The activation of the deltoid muscle showed differences according to the weighted ball conditions $(P<0.05)$, with a lower muscle activation when pitching with an $\mathrm{OB}$ than with an $\mathrm{RB}$ during WU $(P<0.05)$. The latissimus dorsi muscle showed a lower activation when pitching with an $\mathrm{OB}$ than with an $\mathrm{LB}(P<0.001)$ or $\mathrm{RB}(P<0.01)$ during $\mathrm{WU}$, while also showing significant differences according to time $(P<0.05)$. The triceps brachii muscle also showed a lower activation when pitching with an $\mathrm{OB}$ than with an $\mathrm{LB}(P<0.01)$ or $\mathrm{RB}(P<0.05)$ during $\mathrm{WU}$, while also showing significant differences according to time $(P<0.01)$ (Table 5).

\section{Correlation between the muscle activation and ball velocity}

To determine the correlation between the muscle activation and ball velocity, the highest ball velocity and highest muscle activation of each muscle throughout all phases were analyzed. The ball velocity showed significant positive correlations with the trapezius, deltoid, latissimus dorsi, and triceps brachii muscles $(P<0.05)$ (Table 6).

\section{DISCUSSION}

In baseball, pitching is a very complex motion that requires flexibility, muscle strength, coordination, synchrony of muscle firing, and muscle nerve efficiency. During a pitching motion, excessive load is generated on the shoulder joint because enough flexibility for the throwing motion to perform external rotation is required, together with enough stability to prevent dislocation of the shoulder joint (Ouellette et al., 2008). In particular, overhand pitching motions involve extreme recruitment of complex muscles in the shoulder joint to provide functional stability because the shoulder muscles are required to provide a strong force to wrist and the arm acceleration (Collins and Comstock, 2008) and neuromuscular efficiency that can provide stability for proper motional function (Wilk et al., 2000).

Relative to the shoulder joint, a pitching motion can be divided into the wind-up, stride, arm cocking, acceleration, and deceleration phases. In the stride phase, motions, such as shoulder abduction, lateral rotation, and horizontal abduction, occur (Meister, 2000). In the stride phase of this study, muscle activation according to the weight of the ball showed the supraspinatus muscle having a high activation when pitching with an $\mathrm{OB}$. The abduction angle in the pitching shoulder at the initial moment when the foot touches the ground has been reported to be $\sim 80^{\circ}-100^{\circ}$, and at this time, the deltoid and supraspinatus muscles become active to maintain abduction while also maintaining the glenohumeral head (Bradley and Tibone, 1991; Meister, 2000). Therefore, it is believed that the supraspinatus muscle showed a high activation, since it is involved in shoulder abduction to withstand the weight of the heavier ball during pitching with an OB.

As the transition to the arm cocking phase takes place and the shoulder is in its maximum external rotation position, the arm is located slightly behind the torso; here, the posterior deltoid, latissimus dorsi, pectoralis minor, and infraspinatus muscles are responsible for shoulder external rotation (Wilk et al., 2000). Moreover, the serratus anterior muscle becomes most active, as it plays a role in the stabilization and forward traction of the scapula, enabling the scapula to move together with the horizontal adduction of the upper arm (Digiovine, 1992). In the arm cocking phase during WU, the deltoid muscle activation was lower when pitching with an $\mathrm{LB}$ than with an $\mathrm{RB}$, while the biceps muscle showed a lower activation when pitching with an $\mathrm{OB}$ than with an $\mathrm{RB}(P<0.05)$. However, during TBME subsequent to pitching with an $\mathrm{OB}$ during $\mathrm{WU}$, the activation of the latissimus dorsi and biceps brachii muscles significantly increased $(P<0.05)$. A greater maximum shoulder external rotation (MSER) in the throwing arm during the arm cocking phase increases the throwing velocity (Matsuo et al., 2001). Although there is no difference in the MSER when pitching with an LB ( 4 oz) and RB (5 oz) (Fleisig et al., 2006), the increase in the weight of the baseball decreases the MSER. Consequently, it becomes difficult for the shoulder to generate a high torque (Fleisig et al., 1996), and while the shoulder horizontal adduction torque, elbow varus torque, and angular velocities of the shoulder do not show differences when pitching with an LB and $\mathrm{RB}$, they show significant decreases when pitching with an OB (Fleisig et al., 2017). Therefore, pitching with an OB during 
WU decreased the MSER and angular velocities of the shoulder, which resulted in lower muscle activation; however, the muscle activation increased during TBME since an RB was used.

Werner et al. (1993) reported that an internal rotational force of $111 \mathrm{Nm}$ was generated under MSER when transitioning from the arm cocking phase to the acceleration phase, during which time the highest levels of muscle activation were found. In the arm acceleration phase, humeral abduction, horizontal abduction, and internal rotation occurred at a rate of $7,000^{\circ} / \mathrm{sec}$ and pressure of $800 \mathrm{~N}$ (Meister, 2000; Ouellette et al., 2008), where the activation of the pectoralis major, latissimus dorsi, and serratus anterior muscles increased (Harryman et al., 1990). In this study, a higher activation in the upper trapezius, supraspinatus, deltoid, latissimus dorsi, and triceps brachii muscles was found when pitching with an LB and RB during WU, while the activation of the infraspinatus and serratus anterior muscles increased when pitching with an $\mathrm{OB}$. Moreover, the activation of the latissimus dorsi muscle significantly increased during TBME subsequent to pitching with an LB and OB during WU. Such results are similar to those of a report that indicated that pitching with an LB increased the arm swing velocity by increasing the elbow and shoulder velocities and that pitching with an LB during WU increased the muscle activation by increasing the arm velocity (Fleisig et al., 2017; Van den Tillaar and Ettema, 2011; Wang et al., 1995). Moreover, the shoulder internal rotation velocity has been reported to have a direct relationship with the ball velocity, and the ball velocity being higher when pitching with an $\mathrm{LB}$ and $\mathrm{RB}$ than with an $\mathrm{OB}$ during WU may be attributed to differences in the arm velocity (Matsuo et al., 2001).

However, the ball velocity during TBME with an RB subsequent to WU did not show significant differences according to the weight of the baseball; thus, using weighted baseballs does not appear to have a significant acute effect on the ball velocity. Activation in the upper trapezius, supraspinatus, deltoid, latissimus dorsi, and triceps brachii muscles increased after pitching with an $\mathrm{OB}$ during WU to show no difference in the muscle activation during TBME; the activation of these muscles is believed to increase to compensate for the increase in the ball velocity that decreased after pitching with an OB. In particular, as indicated in a report that the muscular strength increased after ballistic training using an OB (Chiang et al., 2010) and using heavy ball holds is a good exercise for increasing biceps muscle strength since they increase elbow flexion torque while significantly reducing elbow torque (Fleisig et al., 2017), the acute use of an OB did not affect the ball velocity. However, continued training may be effective in increasing the ball velocity by activating the agonistic muscles of arm acceleration. Moreover, pitching with an LB increases the arm swing velocity, where high-velocity arm swing movements increase the involvement of fast-twitch muscles to increase the recruitment of high-threshold motor units, which has been suggested to improve explosive force production (Sale, 1987; Smith et al., 1980). Accordingly, continued training using an LB may also serve as one of the methods for increasing the throwing velocity; future studies are needed to observe changes through continued training.

Taken together, the LB and RB showed a small difference in the muscle activation during WU as compared with those during TBME, whereas pitching with an OB showed either an increase or decrease in the muscle activation. However, the muscle activation and ball velocity did not show differences when an RB was used during TBME. Therefore, the weight of the baseball used during WU should be selected according to the player's condition. However, as changes in the muscle activation were found according to the weight of the baseball used, applying this to the training program can have an effect on improving the ball velocity.

\section{CONFLICT OF INTEREST}

No potential conflict of interest relevant to this article was reported.

\section{REFERENCES}

Bradley JP, Tibone JE. Electromyographic analysis of muscle action about the shoulder. Clin Sports Med 1991;10:789-805.

Brose DE, Hanson DL. Effects of overload training on velocity and accuracy of throwing. Res Q 1967;38:528-533.

Chiang KD, Liu C, Kao YC. Effects of throwing different weight baseballs on pitching movement and velocity. Chin J Sports Biomech 2010;2:2230.

Collins CL, Comstock RD. Epidemiological features of high school baseball injuries in the United States, 2005-2007. Pediatrics 2008;121:11811187.

Cram JR, Kasman GS. Holtz J. Introduction to surface electromyography. Maryland: Aspen; 1998.

Cressey E. Weighted baseballs: safe and effective, or stupid and dangerous? [Internet]. Cressey Training Systems, LLC; 2009 Dec 15 [cited 2018 Jan 15]. Available from: http://www.ericcressey.com/weighted-baseballs-safe-and-effective-orstupid-and-dangerous.

DeRenne C, Buxton BP, Hetzler RK, Ho KW. Effects of under- and over- 
weighed implement training on pitching velocity. J Strength Cond Res 1994;8:247-250.

DeRenne C, Ho K, Blitzblau A. Effect of weighted implement training on throwing velocity. J Appl Sports Sci Res 1990;4:16-19.

DeRenne C, Szymanski DJ. Effects of baseball weighted implement training: a brief review. Strength Cond J 2009;31:30-37.

DeRenne C, Tracy R, Dunn-Rankin P. Increasing throwing velocity. Athlet J 1985;65:36-39.

Digiovine NM, Jobe FW, Pink M, Perry J. An electromyographic analysis of the upper extremity in pitching. J Shoulder Elbow Surg 1992;1:1525.

DriveLine Baseball. 4 Great reasons to throw weighted (and lightweight) baseballs [Internet]. Kent (WA): DriveLine Baseball; 2011 Jun 28 [cited 2018 Jan 15]. Available from: http://www.drivelinebaseball.com/4-greatreasons-tothrow-weighted-and-lightweight-baseballs/.

Escamilla RF, Fleisig GS, Barrentine SW, Zheng N, Andrews JR. Kinematic comparisons of throwing different types of baseball pitches. J Appl Biomech 1998;14:1-23.

Escamilla RF, Speer KP, Fleisig GS, Barrentine SW, Andrews JR. Effects of throwing overweight and underweight baseballs on throwing velocity and accuracy. Sports Med 2000;29:259-272.

Fleisig GS, Diffendaffer AZ, Aune KT, Ivey B, Laughlin WA. Biomechanical analysis of weighted-ball exercises for baseball pitchers. Sports Health 2017;9:210-215.

Fleisig GS, Escamilla RF, Andrews JR, Matsuo T, Satterwhite Y, Barrentine SW. Kinematic and kinetic comparison between baseball pitching and football passing. J Appl Biomech 1996;12:207-224.

Fleisig GS, Kingsley DS, Loftice JW, Dinnen KP, Ranganathan R, Dun S, Escamilla RF, Andrews JR. Kinetic comparison among the fastball, curveball, change-up, and slider in collegiate baseball pitchers. Am J Sports Med 2006;34:423-430.

Harryman DT 2nd, Sidles JA, Clark JM, McQuade KJ, Gibb TD, Matsen FA 3rd. Translation of the humeral head on the glenoid with passive glenohumeral motion. J Bone Joint Surg Am 1990;72:1334-1343.

House T. Velocity+arm care. Elizabethtown (KY): The Player's Dugout; 2016 May 1 [cited 2018 Jan 15]. Available from: http://www.velocityplusarmcare.com.
Matsuo T, Escamilla RF, Fleisig GS, Barrentine SW, Andrews JR. Comparison of kinematic and temporal parameters between different pitch velocity groups. J Appl Biomech 2001;17:1-13.

Meister K. Injuries to the shoulder in the throwing athlete. Part one: Biomechanics/pathophysiology/classification of injury. Am J Sports Med 2000;28:265-275.

Ouellette H, Labis J, Bredella M, Palmer WE, Sheah K, Torriani M. Spectrum of shoulder injuries in the baseball pitcher. Skeletal Radiol 2008; 37:491-498.

Pavlovich L. Weighted ball training started years ago. Collegiate Baseball 2014;4:8.

Potteiger JA, Blessing DL, Wilson GD. Relationship between pitching velocity, anaerobic power and lean body mass. J Appl Sports Sci Res 1989; 133:74.

Sale DG. Influence of exercise and training on motor unit activation. Exerc Sport Sci Rev 1987;15:95-151.

Smith JL, Betts B, Edgerton VR, Zernicke RF. Rapid ankle extension during paw shakes: selective recruitment of fast ankle extensors. J Neurophysiol 1980;43:612-620.

van den Tillaar R, Ettema G. A comparison of kinematics between overarm throwing with $20 \%$ underweight, regular, and 20\% overweight balls. J Appl Biomech 2011;27:252-257.

Van Huss WD, Albrecht L, Nelson R, Hagerman R. Effect of overload warm-up on the velocity and accuracy of throwing. Res Q Am Assoc Health Phys Educ Recreat 2013;33:472-475.

Wang YT, Ford HT 3rd, Ford HT Jr, Shin DM. Three-dimensional kinematic analysis of baseball pitching in acceleration phase. Percept Mot Skills 1995;80:43-48.

Werner SL, Fleisig GS, Dillman CJ, Andrews JR. Biomechanics of the elbow during baseball pitching. J Orthop Sports Phys Ther 1993;17:274278.

Wilk KE, Mesiter K, Fleisig G, Andrews JR. Biomechanis of the overhead throwing motion. Sports Med Arthrosc Rev 2000;8:124-134.

Yang WW, Liu YC, Lu LC, Chang HY, Chou PP, Liu C. Performance enhancement among adolescent players after 10 weeks of pitching training with appropriate baseball weights. J Strength Cond Res 2013;27: 3245-3251. 